\title{
Use of NDT Inspection Data to Improve Rail Damage Prediction Models
}

\author{
Pelin Boyacioglu*, Adam Bevan*, Andy Vickerstaff ${ }^{\dagger}$ \\ *Institute of Railway Research, University of Huddersfield, UK, p.boyacioglu@hud.ac.uk, \\ ${ }^{+}$London Underground, UK
}

Keywords: rolling contact fatigue, non-destructive testing, rail inspection, RCF damage modelling.

\begin{abstract}
Infrastructure managers (IMs) endeavour to eliminate rail defects at an early stage since they impact on safety and quality of operation and increase system costs. London Underground (LUL) uses several non-destructive testing (NDT) techniques in rail inspection to detect the emerging defects and monitor the growth of previously recorded defects. This task mainly aims to prioritise maintenance and renewal activities and record their completion. However, when the high traffic demand and limited maintenance periods are considered, these requirements bring additional pressures to the maintenance team. To optimise maintenance planning, sufficient and reliable field data along with accurate damage prediction are required. Recent developments in NDT technology has seen the introduction of devices to measure crack depth which is a key parameter in the assessment of crack severity and rail life. Therefore, contrary to previous research which mainly utilised observations of rail surface condition, the use of new NDT techniques can support the development and validation of new rail damage models which will help to improve maintenance planning and move to condition-based maintenance strategy.
\end{abstract}

\section{Introduction}

Safety and reliability of rails primarily depend on detection, monitoring and maintenance of rolling contact fatigue (RCF) defects. Since when they are undetected and untreated, they can further propagate and generate potential for rail failures. Therefore, infrastructure managers (IMs) tend to detect these cracks at an early stage in order to reduce the risk of failure as shown in Figure 1. However, in order to prevent premature rail replacements and unnecessary maintenance costs, it is important to obtain reliable crack depths measurements and manage crack growth. Thus, in addition to early detection, optimum maintenance strategy should be developed to balance all the negative consequences. Rail grinding, in a similar manner to wear, is an effective maintenance method to remove and/or truncate the initiated cracks. In railways, it is generally carried out as a preventive maintenance regime which divided into fixed-interval maintenance (cyclic) and condition-based maintenance [1]. London Underground (LUL) often conducts fixed-interval maintenance based on a 'track loading factor'. This is calculated considering the tonnage and running speed of each of the line sections [2].

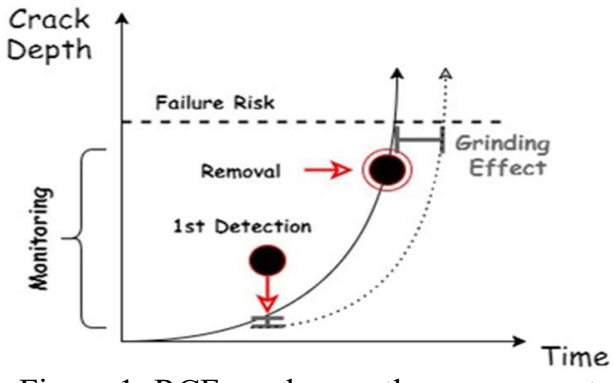

Figure 1: RCF crack growth management

Due to high traffic demand and limited maintenance periods, setting a single frequency is seemed to be more suitable. However, an ideal solution is to implement condition-based maintenance. Nonetheless, the challenges in RCF management makes it harder to apply this regime. To determine the next interval, reliable and sufficient field data with an accurate damage prediction model are required. Hence, monitoring of defects particularly on the study network is an essential part to conduct this maintenance regime, as it helps to understand the existing RCF mechanism in rails and to support the damage prediction modelling studies. Nevertheless, limitations in rail inspection devices sometimes reduce the reliability of field defect data and the changing operational and environmental conditions in rail traffic make it difficult to monitor the crack propagation from field observations. In addition, the complex crack growth mechanism affects the rate of propagation and therefore, the depth of each crack varies based on its development path and initiation angle from the surface.

In this study, the rail inspection in LUL is explained by providing the different non-destructive testing (NDT) techniques used on the lines. Their efficiency was evaluated and the measurement outputs were compared on the selected two metro lines: Bakerloo and Jubilee. Moreover, the study utilized these outputs to validate the current rail damage prediction model. The field defect data particularly the MRX Rail Surface Crack Measurement (MRX-RSCM) crack depth measurements were correlated with model results. As a result, a new RCF crack depth prediction model was developed which may help to better predict the future RCF severity conditions and help to change from fixed-interval maintenance to condition-based maintenance regime on LUL.

\section{Rail Inspection in London Underground}

LUL currently uses NDT devices, such as ultrasonic and MRXRSCM sensors to detect defects and their severity. In addition, 
visual inspections are also carried out to verify these measurements and particularly, to identify defects which may have been missed or rectified and to define potential risks to generate rail failures.

\subsection{Ultrasonic inspection}

Ultrasonic inspection is mainly performed by the portable detectors. A beam of ultrasonic energy generated by a piezoelectric element is transmitted into the rail and the reflected energy of the transmitted beam is then detected using a collection of transducers. The sensor analyses this energy and identifies defects from the disturbances in the signal [3]. Although previous studies mentioned that the reliability of its outputs were sometimes reduced in detecting surface-initiated cracks $(<4 \mathrm{~mm})$ and cannot accurately detect critical defects masked by spalled rail or shallow defects, the detector with multiple transducers $\left(70^{\circ}\right)$ was employed to increase the detection ability as shown in Figure 2. This also enable the detection of defects in the rail web.

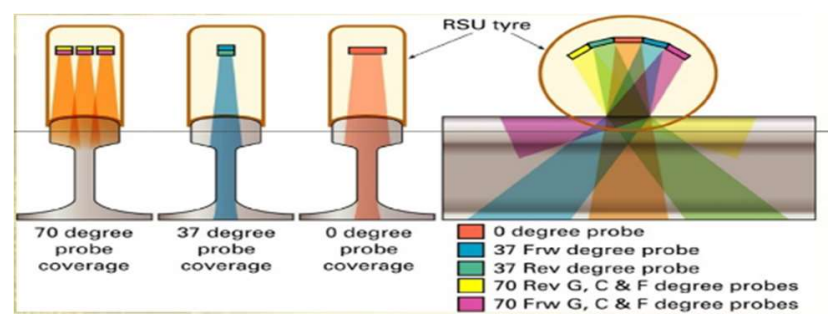

Figure 2: Ultrasonic transducers at various angles [3]

\subsection{Visual inspection}

Visual inspection is mainly carried out in the form of patrolling. In this method, the crack severity classification is generally made based on the relationship given in Figure 3. To understand the correlation between surface length and depth of a crack, the study sectioned a large number of rail samples and examined RCF cracks [4]. It was found that if the surface length of a crack is larger than $20 \mathrm{~mm}$, it corresponds to heavy severity and the risk of the crack growing further rapidly increases. LUL have added an additional severe classification (highlighted in red colour in Figure 3), which indicates that the depth is $>7 \mathrm{~mm}[2]$.

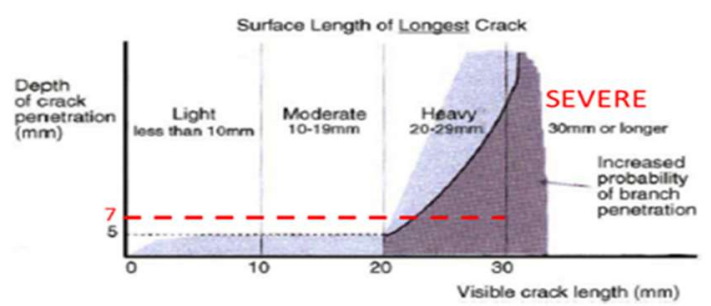

Figure 3: Crack severity assessment [4]

However, the recent studies have demonstrated that there was no direct correlation, as the position of the rail (e.g: whether it is on curved, transition or straight track) along with the different rail steel compositions changed the crack growth angle, leading to different crack depths regardless of its surface length [5]. Besides these deficiencies in the crack severity (depth) estimation, the visual inspection can contain some errors resulting from misinterpretation of the field conditions. For instance, it was mentioned in one of the studies that poor sight conditions in tunnels and accumulated lubricants particularly on the high (outer) rails of curved tracks might cause misleading records [6]. Moreover, the reflections of the sun and raindrops may also influence visibility and mask small defects on the open track sections. Moderate and severe squats are sometimes reported as wheelburn defects in which the previous related study and data analysis in the next step demonstrated the vice versa on LUL.

In addition to conventional visual inspection method, multiple trains fitted with Automatic Track Monitoring System (ATMS) which provides the condition of rail head surface using video imaging [2]. But, it should be also noted that due to movement of cameras, some of the defects may not be efficiently detected and visualized by these systems and it requires additional offline analysis to identify defective areas.

\subsection{MRX-RSCM inspection}

Even though the estimation of crack depth from observed surface length could be misleading, it is a crucial feature in the assessment of rail residual life and maintenance planning. To increase the accuracy of this data and to adopt an advanced inspection technique in the rail monitoring, LUL started to use magnetic flux leakage based sensor MRX-RSCM (developed by MRX Technologies). It can detect both surface and subsurface RCF damage with a maximum of $7 \mathrm{~mm}$ crack depth by measuring the changes in the flux strength induced by a magnet located in the device. When there are no defects, the flux lines travel undisturbed through the railhead, but in rails with defects, the flux cannot travel as easily and some flux leaks [8]. Additionally, it gives information about the railhead surface condition and location of defects. With the help of 19 sensors positioned to $5 \mathrm{~mm}$ apart to each other (Figure 4) and $2.5 \mathrm{~mm}$ longitudinal detection interval, $5 \mathrm{~mm} \times 2.5 \mathrm{~mm}$ grids are generated on top surface demonstrating the severity of damage by the help of pre-defined colour scale.

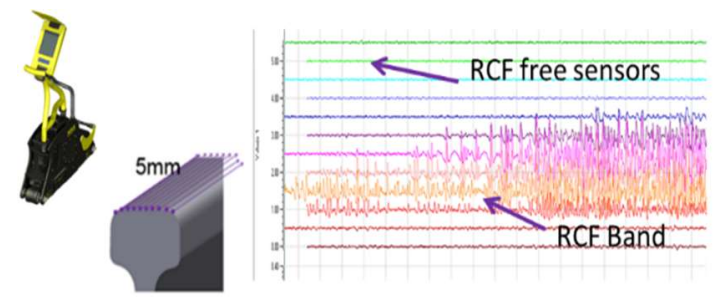

Figure 4: Sensors ' positions and measurements [7]

To validate the MRX-RSCM measurements, a study conducted a number of examinations which found that the system provided a good correlation for the cracks between $1.5 \mathrm{~mm}$ and $5 \mathrm{~mm}$ depth and classified as severe damage when there was spalling of material [8]. In addition, the weld locations were accurately distinguished. However, the further studies which were carried out under International Collaborative Research Initiative (ICRI) project revealed significant differences between the crack depth measurements using the MRX-RSCM and Draisine systems and rail sectioning results. After measurements of cracks in the specified regions, the rails were 
sectioned and the depths of cracks were analysed under the electron microscope. It was found that Draisine which uses the eddy current technique measured the crack length and calculated the crack depth based on assumed $25^{\circ}$ crack angle overestimated the damage depth when the actual angles were smaller. Similarly, the MRX-RSCM also provided unsatisfactory validation as it measured relatively higher than the actual crack depth. Nonetheless, it was mentioned that the $1 \mathrm{~m}$ measurement output interval may be responsible for this inaccuracy, as the length of rail samples were shorter [9].

\section{Field defect data analysis}

Although each rail inspection system is employed for different reasons, their efficiency was evaluated by comparing defects reported on the data sheets (ultrasonic and visual inspection) and measured by the MRX-RSCM device on the Bakerloo and Jubilee lines.

The defects are recorded in the rail defect form which includes the information such as date and type of inspection, rail defect type (code number), severity, location, repair/maintenance technique and the minimum actions which have to be taken before its removal. Depending on the severity of crack and risk level, the type of repair or maintenance was determined from the LUL's standards. For example, while the low-priority (depth $<5 \mathrm{~mm}$ ) defects are recommended for planned monitoring and maintenance, such as grinding, minimal repair and rail welding, the high-priority defects (depth $\geq 5 \mathrm{~mm}$ ) require a more rapid rail replacement with minimum actions such as speed limits and emergency clamps in the highly critical defect [2].

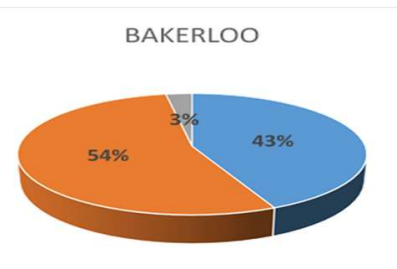

= Visual $=$ Ultrasonic $=$ MRX-RSCM

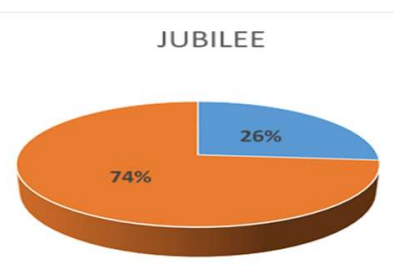

= Visual $=$ Ultrasonic
Figure 5: Rail inspection methods in defect data sheet

The defect reports are listed in defect data sheets for each railway line on LUL. In this study, the data was evaluated for the years 2013-2015. Figure 5 displays the percentage of each inspection method reported in the defect data sheets. As it can be seen, the ultrasonic testing was the primary technique for both of the lines. However, when the defect notes were particularly examined, it was noted that approximately $25 \%$ and $5 \%$ of the total defects on Bakerloo and Jubilee lines respectively were recorded as ultrasonically untestable as the surface damage prevented the testing of the detector. Therefore, under this circumstance, visual inspection played a key role as nearly half of the listed defects in the Bakerloo line were reported during site visits. On the other hand, whereas MRX-RSCM data were considered in the Bakerloo line, it was not listed in the defect data sheets of the Jubilee line. The MRX-RSCM measurements conducted in two different time intervals for the similar years 2013-2015. However, when the different outputs were overlaid particularly in the comparison of distances in consecutive MRX-RSCM measurements, several occasional gaps were revealed in the data. Owing to changes in the railhead profile and slipping of the sensor wheels, the detector cannot effectively adjust and produced different distances for the similar track sections.

Figure 6 displays the correlation of (reported) defect data sheet outputs and the 2014-2015 MRX-RSCM crack depth measurements on the right rails of the Bakerloo line. While the blue arrows demonstrate the common locations (solid lines for the defective rails, the dashed lines for the non-defective rails), the orange arrows indicate the unmatched areas. As shown, a number of areas demonstrated good correlations, the transverse $\mathrm{RCF}$ defects which was defined to squat defects (squat with tache ovale-T/O) exceeding critical sizes according to LUL's standard had higher crack depths such as at approx. Ch. 7+000 $\mathrm{km}, \quad$ Ch. $8+000 \mathrm{~km}, \quad$ Ch. $8+500 \mathrm{~km}, \mathrm{Ch} .10+500 \mathrm{~km}$. Additionally, the dashed lines at Ch. $4+500 \mathrm{~km}$ and Ch.7+500 $\mathrm{km}$ presented the areas where all the methods were in agreement about the intact rails. On the other hand, even though MRX-RSCM showed damages at Ch. $4+000 \mathrm{~km}$ and $5+000 \mathrm{~km}$, no defects were reported in the defect forms. There might be several reasons behind these inconsistencies; the measured damage by the sensor could be located inside the railhead (sub-surface) therefore, it could not detect by the other methods. In addition, the aforementioned overestimation of the detector might cause deeper cracks shown in these areas. Moreover, the change in crack depth was noticed. For example, $4 \mathrm{~mm}$ depth at around Ch. $8+000 \mathrm{~km}$ in 2014 was reduced in 2015 due to grinding and/or natural wear. On the other hand, the decrease in crack depth at Ch. $1+000 \mathrm{~km}, \mathrm{Ch} .2+000 \mathrm{~km}$ and Ch. $3+600 \mathrm{~km}$ was caused by the rail replacement.

Similarly, the Jubilee line produced good agreement in several locations due to the higher number of reported defects as shown in Figure 7 (the line distance is also longer). Both shelling and squat defects matched with MRX-RSCM outputs such as at Ch. $5+000 \mathrm{~km}$, Ch. $15+000 \mathrm{~km}$, and Ch. $27+500 \mathrm{~km}$. However, the orange arrows at Ch.10+000 km and Ch. $12+000$ $\mathrm{km}$ again confirmed its overestimation. Nonetheless, it should be also noted that the defect data sheet outputs may contain misleading results. For example, the study which investigated squats on the LUL network found that some of the observed cracks were caused by thermal damages particularly, the limited wheelslip in poor adhesion areas [10]. Therefore, a new name was given and called as studs (squat type of defects). But, although no studs were reported on this line, the LUL staff declared that some of the squats could be studs as shown between Ch.25+000-35+000 km.

In the study, the correlation of several NDT techniques' outputs help to better understand and analyse crack severity on the studied lines. Even though the MRX-RSCM demonstrated certain reductions in the reliability of data stemming from overestimations in crack depth measurements and distance discrepancies, it provided valuable information in the monitoring of defects. For instance, while the defect data sheets provided that the high rails were susceptible to damage, 


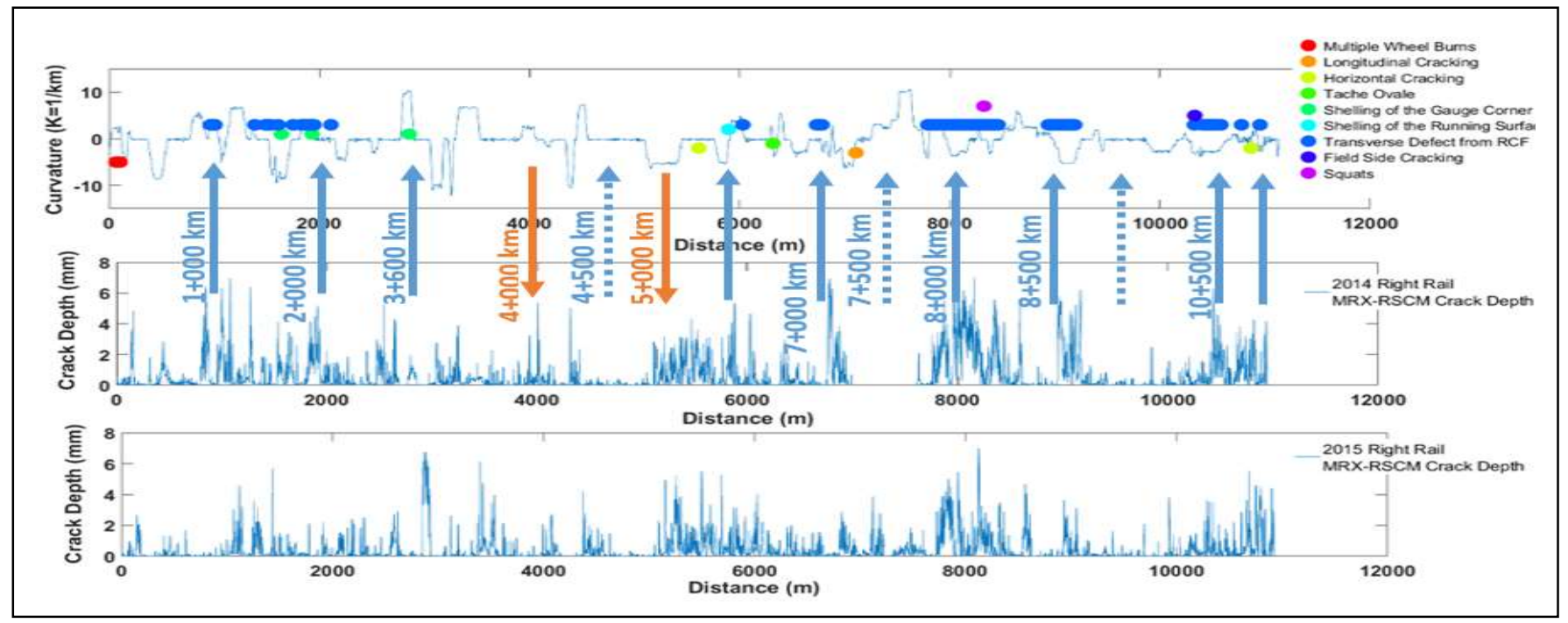

Figure 6: Correlation of (reported) defect data sheet outputs and MRX-RSCM measurements on the right rail of the Bakerloo line

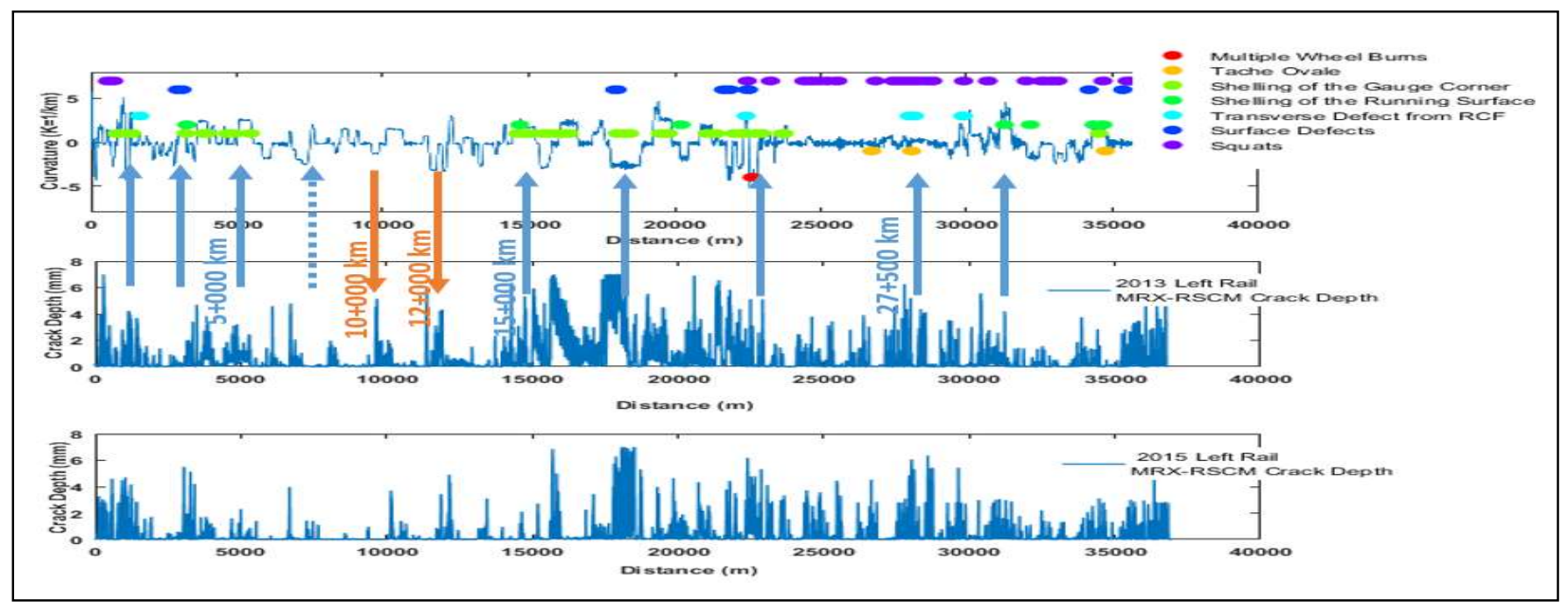

Figure 7: Correlation of (reported) defect data sheet outputs and MRX-RSCM measurements on the left rail of the Jubilee line

the MRX-RSCM showed the severity of cracks on low (inner) rails of curved and tangent track which was also pronounced by the maintenance team on LUL. It should be also noted that correct data interpretation is very significant, as the rail maintenance history data such as rail grinding and replacements were taken into account in the field data analysis.

\section{Rail Damage Predictions}

The rail inspection data were also used to assist the validation of the current damage predictions. The VAMPIRE vehicle dynamics route simulations were conducted for both of the lines to calculate wheel-rail contact forces and the Whole Life Rail Model (WLRM) was utilized to predict the damage. The model uses the parameter Wear Number $(\mathrm{T} \gamma)$ which shows the dissipated energy at the wheel-rail contact. It was computed from the sum of creep forces and creepages for the longitudinal $\left(\mathrm{T}_{\mathrm{x}}, \gamma_{\mathrm{x}}\right)$, lateral $\left(\mathrm{T}_{\mathrm{y}}, \gamma_{\mathrm{y}}\right)$ and spin $\left(\mathrm{M}_{\mathrm{z}}, \mathrm{w}_{\mathrm{z}}\right)$ components.

$$
\mathrm{T} \gamma=\mathrm{T}_{\mathrm{x}} \gamma_{\mathrm{x}}+\mathrm{T}_{\mathrm{y}} \gamma_{\mathrm{y}}+\mathrm{M}_{\mathrm{z}} \omega_{\mathrm{z}}
$$

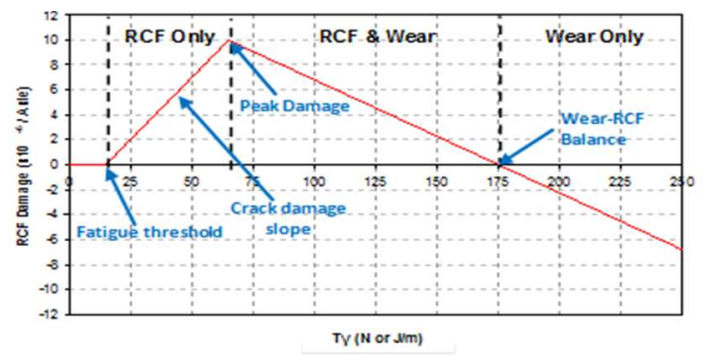

Figure 8: WLRM RCF damage function

As shown in Figure 8, it describes the interaction between wear and RCF and hence, helps to identify the dominant damage mechanism in rails. As the energy increases in the model, the risk of RCF decreases and wear increases (e.g increased wear removes RCF cracking). However, it should be noted that WLRM uses the term 'signed $\mathrm{T} \gamma$. This assumes that rail RCF can only be generated when the wheel-rail creep forces are in the traction direction. Therefore, the model eliminates the braking regions on rail and suggested that this condition will give rise to wheel damage. In the WLRM, the relationship 
between 'signed $\mathrm{T} \gamma$ ' and RCF Damage Function was established through number of laboratory tests and field observations in the GB rail network. This non-dimensional function was particularly linked to the surface observations such as crack length, position and orientation [11]. Contrary to previous studies which mainly considered track and vehicle characteristics of the mainline routes, this study applied the model on the metro-underground systems and used the NDT outputs in the validation. Due to uncertainties in the breaking points such as fatigue threshold and wear-RCF balance, the location of high values of 'signed $\mathrm{T} \gamma$ ' used as an indicator of increased susceptibility to RCF damage and were correlated with the MRX-RSCM crack depth measurements.

Figure 9 displays the comparison of 2014-2015 MRX-RSCM measurements with 'signed T $\gamma$ ' values on the right rail of the Bakerloo line. In case of two-point (tread and flange) contacts which was particularly evident on the outer (high) rails of curved tracks, the $\mathrm{T} \gamma$ values were summed to find the maximum energy produced at that distance on the lines. While the $y$-axis indicates the cant values $( \pm 20 \mathrm{~mm}$ corresponds to straight sections), the colorbar shows the 'signed $\mathrm{T} \gamma$ ' values.
As indicated by the blue arrows, the several locations of high 'signed $\mathrm{T} \gamma$ ' coincided with locations of crack measurements. Similar to previous findings of the model, the high rails on curved tracks such as at Ch. $8+000 \mathrm{~km}$ and Ch.10+500 km provided good agreement since, creep forces were often in the traction direction. But, the check rails which were installed on curves for radius smaller than $200 \mathrm{~m}$ in this line sometimes restricted the level of flange contacts on high rail. Whereas this sometimes gave rise to increased tread contact energy and good correlation such as $\mathrm{Ch} .3+000 \mathrm{~km}$, poor agreement could be also generated such as $\mathrm{Ch} .5+000 \mathrm{~km}$. Generally, the low rails had the highest values Ch. $1+500 \mathrm{~km}$ and Ch.7+500 km. Conversely, the 'signed $\mathrm{T} \gamma$ ' became zero on braking regions and hence, the model would fail to predict the damage as indicated by the orange arrows. For instance, the low rails of shallower $(\mathrm{R}>200 \mathrm{~m})$ curved tracks (e.g: Ch.4+000 km) and tangent track sections (e.g: Ch.11+000 km) showed unsatisfactory correlations.

Since the Jubilee line has no check rails (minimum curve radius $250 \mathrm{~m})$, "signed $\mathrm{T} \gamma$ " was again successful in predicting the locations of most of the high rail RCF as it can be especially

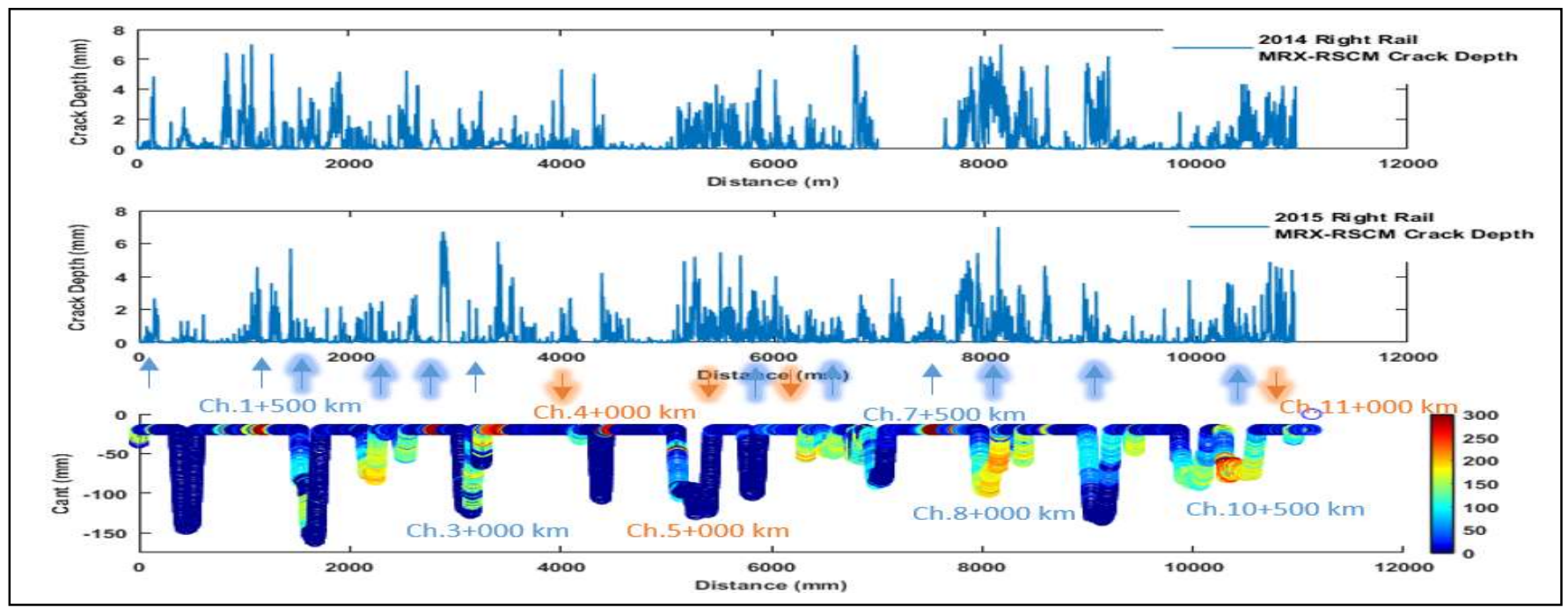

Figure 9: Damage 'signed T $\gamma$ ' predictions and MRX-RSCM measurements on the right rail of the Bakerloo line

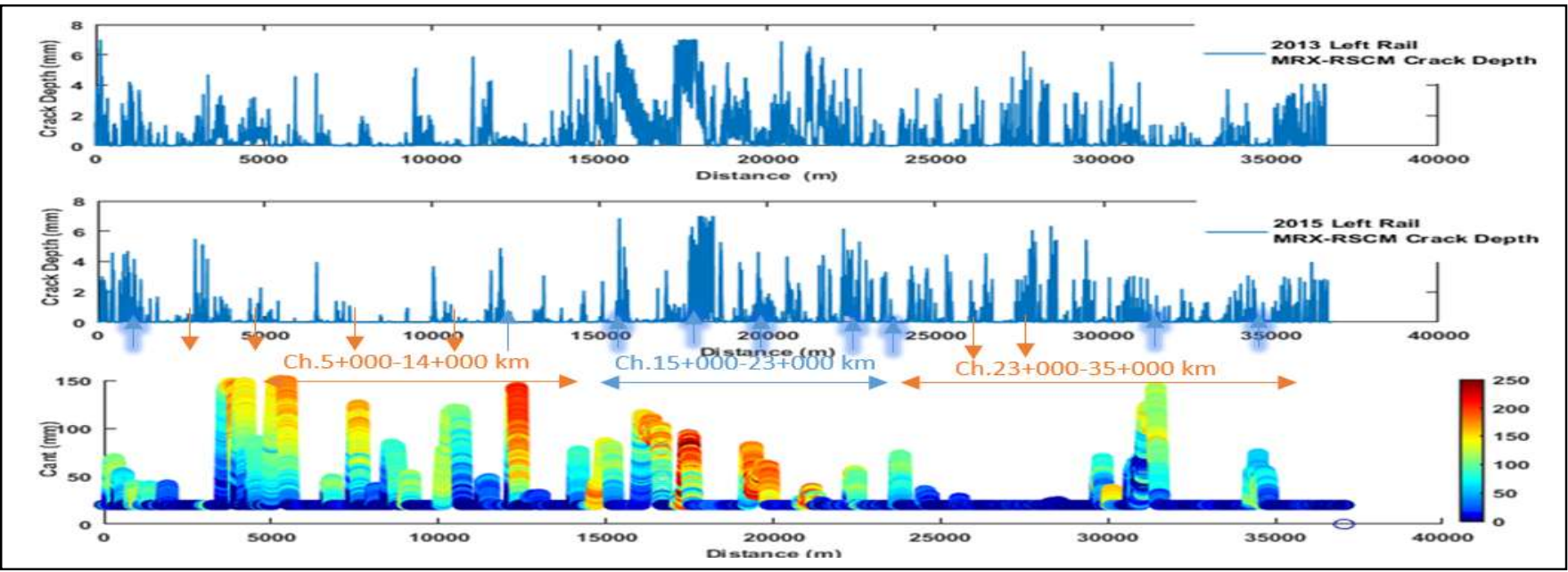

Figure 10: Damage 'signed T $\gamma$ ' predictions and MRX-RSCM measurements on the left rail of the Jubilee line 
seen $($ Ch. $15+000-23+000 \mathrm{~km})$ but, low rails and tangent tracks which were largely measured in the Ch.23+000-35+000 km (outside section) cannot be predicted by $\mathrm{T} \gamma$. Nonetheless, when the possibility of stud (thermal) damage is considered in these areas, the inaccurate prediction might become reasonable as WLRM was developed to predict classic high rail RCF related cracking. On the other hand, the large levels of $\mathrm{T} \gamma$ which were mainly produced on the high rails between Ch.5+000-14+000 $\mathrm{km}$ led to poor agreement with the relatively undamaged area.

As it can be seen, although the high 'signed $T \gamma$ ' values provided good correlations with several measured crack locations, it sometimes overestimated since, no damage was measured in certain locations. However, this might be caused by the differences in rail ages (recent rail replacements) or grinding activities. On the other hand, it was also shown that especially the current assumption of the model (traction direction) led to underestimation at a number of locations. In the study, the key areas which contribute to these deficiencies were identified. To improve the accuracy of damage predictions, the influence of key changes at the wheel-rail contact parameters at the selected RCF sites were further investigated and compared with the conditions on areas where no RCF was measured since, the high energy values may also give rise to higher wear rate which in turn, may remove the initiated cracks. As a result, a new RCF crack depth prediction model was developed which uses the 'raw $\mathrm{T} \gamma$ ' in combination with Shakedown Map. Figure 11 shows the comparison of new RCF crack depths predictions with MRX-RSCM measurements on the low rail RCF site in the Bakerloo line. Whilst the new model again produced over-predictions, the difference was under $0.5 \mathrm{~mm}$ levels.

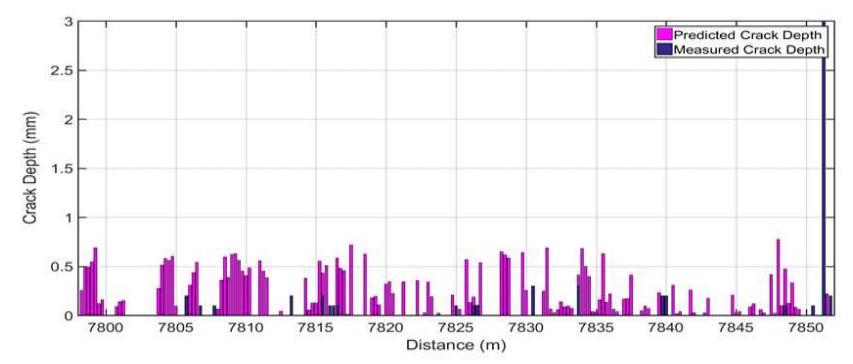

Figure 11: New RCF crack depth predictions on the low rail $\mathrm{RCF}$ site in the Bakerloo line

\section{Conclusions}

In this study, it was suggested that reliable and sufficient field monitoring defect data is essential to understand the damaging conditions and to increase the accuracy of current prediction models. On LUL, several different NDT techniques have been employed. This study evaluated their efficiency by comparing their measurement outputs on the Bakerloo and Jubilee lines. It was noted that the reliability of the field data was sometimes reduced from various factors. However, to increase the reliability in defect monitoring and measure crack depth, MRX-RSCM device was utilized. Contrary to previous studies, this provided an opportunity to correlate damage predictions with the NDT data. Whilst the 'signed $T \gamma$ ' successfully predicted the locations of certain damage locations measured by MRX-RSCM, there were several sections which had poor agreement. These inaccuracies were potentially caused by the limitations and assumptions used both in the inspection data and the model. Therefore, further investigations at selected RCF sites were conducted to understand the changes and improve the accuracy of the modelling. The crack growth from consecutive MRX-RSCM data was analysed and the interaction of wear was considered. Additionally, $1 \mathrm{~m} \mathrm{MRX-}$ RSCM measurement output interval was reduced to $250 \mathrm{~mm}$ to prevent the overestimations in the inspection data. As a result, a new RCF crack depth prediction model was developed in the study. The future research will present the performance of this new model under various real track conditions. With respect to increased accuracy and crack depth estimations, it is suggested that maintenance planning can be optimised and conditionbased maintenance strategy can be implemented.

\section{Acknowledgements}

Special thanks to MRX technologies Karl Gammon, Dr. Stephanie Klecha and for their collaboration.

\section{References}

[1] S. Kumar, U. Espling, and U. Kumar. "Holistic procedure for rail maintenance in Sweden", Proc. IMechE Part F: Journal of Rail and Rapid Transit 222.4: 331-344 (2008).

[2] London Underground, "S1158 Track Inspection and Maintenance Standard”, London, (2013).

[3] M. P. Papaelias., C. Roberts, and C. L. Davis. "A review on non-destructive evaluation of rails: state-of-the-art and future development", Proc. IMechE Part F: Journal of Rail and Rapid Transit 222.4:367-384, (2008)

[4] W. E. Glavin, J. I. Aspebakken, and G. O. Besch. "Heavy haul:Burlington Northern perspective", Fourth International Heavy Haul Railway Conference 1989: Railways in Action, The. Institution of Engineers, Australia, (1989).

[5] Innotrack, "D4.1.4: Rail degradation algorithms" Tech. report, Innovative Track Systems, Project no. TIP5-CT-2006031415O. International Union of Railways (UIC), (2009).

[6] Z. Popović, L. Lazarević, L. J. Brajović, "Managing rail service life", Metalurgija, 53(4),721-724, (2014).

[7] MRX Technologies, "Rail surface Crack Measurement Manual", Australia, (2011).

[8] S. Kaewunruen, "Identification and prioritization of rail squat defects in the field using rail magnetisation technology." Proc. of International Society for Optics and Photonics, (2015).

[9] E. Magel, "Eloctromagnetic measurement of rail surface cracking”, WRI Heavy Haul Seminar, (2015).

[10] S. L. Grassie, "Studs and squats: The evolving story", Wear (2016).

[11] M. Burstow, "Whole life rail model application and development-Continued development of an RCF damage parameter", RSBB T115 project report, (2004). 